\title{
Serious cervical hematoma after stellate ganglion block
}

\author{
Tomohisa Uchida $\cdot$ Shinichi Nakao $\cdot$ \\ Masahiro Morimoto • Tatsushige Iwamoto
}

Received: 24 January 2014/ Accepted: 30 August 2014/Published online: 26 September 2014

(C) Japanese Society of Anesthesiologists 2014

Keywords Stellate ganglion block (SGB) - Cervical hematoma $\cdot$ Venous bleeding

To the Editor:

Stellate ganglion block (SGB) is widely used to treat upper-extremity and facial pain. Cervical hematoma is one of the most serious and sometimes life-threatening complications of SGB. Here, we report a case of life-threatening neck and mediastinal hematoma due to venous bleeding $2 \mathrm{~h}$ after SGB in a patient without hemostatic abnormalities or receiving anticoagulants and/or antiplatelet drugs.

A 44-year-old man sensed a persistent pain and numbness of the lower left lip after treatment in a dental clinic. Under a diagnosis of neuropathic pain, we administered SGB and pregabalin at $150 \mathrm{mg} /$ day. Two hours after SGB, however, he presented to our hospital complaining of severe dyspnea and pain in the neck and back and showed slight cervical swelling and hypoxemia. We tried to intubate but failed because of severe upper airway edema and performed an emergency tracheostomy in the operating room. Careful observation of the SGB puncture site revealed a massive cervical hematoma, and we removed it and ligated the vein responsible for the bleeding.

Higa et al. [1] reviewed clinical reports on cervical hematomas after SGB and reported that the most frequent initial symptoms are neck pain, dyspnea, and neck swelling. In our case, although the patient also had neck pain and dyspnea, cervical swelling was not so marked, probably because bleeding was venous. We assume the reason bleeding was prolonged and aggravated was the possibility that we tore a vascular wall, especially when pulling out a needle, because the tip may have been bent like a hook while advancing it and pressing it hard on the bone surface. Ultrasound guidance for SGB seems to be a good choice to avoid hematoma formation.

Conflict of interest None.

\section{Reference}

1. Higa K, Hirata K, Hirota K, Nitahara K, Shono S. Retropharyngeal hematoma after stellate ganglion block: analysis of 27 patients reported in the literature. Anesthesiology. 2006;105:1238-45.
T. Uchida $(\bowtie) \cdot S$. Nakao $\cdot$ M. Morimoto $\cdot$ T. Iwamoto Department of Anesthesiology, Kinki University Faculty of Medicine, 377-2, Ohno-Higashi, Osakasayama, Osaka 589-8511, Japan

e-mail: anesth@med.kindai.ac.jp 